\title{
Craneosinostosis, una perspectiva pediátrica
}

\author{
Craniosynostosis, a pediatric perspective
}

\author{
José Pablo Fernández V. ${ }^{\text {a }}$, Gabriela Chica H. ${ }^{\mathrm{b}}$, Andrés Goycoolea R. ${ }^{\mathrm{c}}$
}

aServicio de Neurocirugía Infantil, Instituto de Neurocirugía Asenjo. Santiago, Chile. bPrograma de Título de Especialidad en Neurocirugía, Universidad de Chile, Santiago, Chile.

‘Servicio de Neurocirugía Infantil, Instituto de Neurocirugía Asenjo. Santiago, Chile.

Recibido: 23 de octubre de 2019; Aceptado: 19 de mayo de 2020

\section{¿Qué se sabe del tema que trata este estudio?}

Es una patología poco frecuente, que requiere un alto nivel de sospecha para su derivación precoz. La forma del cráneo debe ser evaluada por el pediatra en cada control sano, evaluando dirigidamente la forma.

\section{¿Qué aporta este estudio a lo ya conocido?}

Describe las características fisiopatológicas de esta enfermedad, su clasificación y entrega recomendaciones sobre cuando derivar.

\section{Resumen}

La craneosinostosis se define como el cierre prematuro de una o más suturas del cráneo, que se manifiesta por una forma anormal de la cabeza. Es una condición infrecuente, pero requiere ser reconocida y derivada oportunamente a Neurocirugía para prevenir complicaciones. El objetivo de esta revisión es describir las características clínicas y genéticas más frecuentes de esta patología, su clasificación de acuerdo a la forma del cráneo y los signos más característicos para lograr reconocerla oportunamente. Se realizó una búsqueda de artículos científicos en bases de datos Pubmed, Scielo y EMBASE con las palabras craneosinostosis, plagio, escafo y braquicefalia. Se seleccionaron artículos en español e inglés que describieran las características de la patología y su manejo, optando por revisiones sistemáticas o recomendaciones de sociedades científicas cuando estuvieran disponibles. La craneosinostosis puede presentarse en forma aislada o asociada a otras deformidades. Su clasificación depende de la(s) sutura(s) afectada(s), lo que lleva a la forma característica del cráneo y de la presencia de otras malformaciones. Suele diagnosticarse y derivarse de forma tardía, lo que se asocia a complicaciones como hipertensión endocraneana y alteración del desarrollo encefálico. La cirugía precoz tiene menor comorbilidad y mejores resultados estético. En conclusión, la forma anormal del cráneo debe hacer sospechar la presencia de craneosinostosis, aunque se presente en forma aislada. El manejo quirúrgico antes del año de vida se asocia a mejor pronóstico.
Palabras clave: Craneosinostosis; Plagiocefalia; Escafocefalia; Braquicefalia 


\begin{abstract}
Craniosynostosis is defined as the premature fusion of one or more skull sutures, characterized by an abnormal shape of the head. It is a rare condition but should be recognized and timely referred to Neurosurgery in order to prevent complications. The objective of this review is to describe the most frequent clinical and genetic characteristics of this pathology, its classification according to the shape of the skull, and the most characteristic signs to achieve timely recognition. A search for scientific articles in Pubmed, Scielo, and EMBASE databases was performed using the terms craniosynostosis, plagiocephaly, scaphocephaly, and brachycephaly. We selected articles in Spanish and English that described the characteristics of the pathology and about its management, choosing systematic reviews or recommendations from scientific societies when available. Craniosynostosis may occur in isolation or associated with other deformities. Its classification depends on the affected suture(s), leading to the characteristic shape of the skull and the presence of other malformations. This condition is usually diagnosed and referred late, which is associated with complications such as intracranial hypertension and impaired brain development. Early surgery has less comorbidity and better esthetic results. In conclusion, the abnormal shape of the skull must raise the suspicion of craniosynostosis, even if it occurs in isolation. Surgical management before one year of life is associated with a better prognosis.
\end{abstract}

\section{Keywords:}

Craniosynostosis;

Plagiocephaly;

Scaphocephaly;

Brachycephaly

\section{Introducción}

La craneosinostosis corresponde al cierre prematuro de 1 o más suturas craneales ${ }^{1}$. El término craneoestenosis, aunque utilizado como sinónimo en ocasiones, se refiere al conflicto de espacio que puede ser secundario a una craneosinostosis ${ }^{2}$. Se estima una frecuencia de 1 cada 2000-2500 RNV ${ }^{3}$, siendo más frecuente en hombres. Lamentablemente no se cuentan con datos nacionales, pero en el Instituto de Neurocirugía, centro de referencia de esta patología en el sistema público, se operaron 52 casos el año 2018 (información no publicada).

Las suturas craneales separan las placas óseas del cráneo y permiten el rápido crecimiento de este en los primeros 2 años de vida, el que es secundario al crecimiento del cerebro. La fusión prematura de las suturas craneales, impide el crecimiento normal del cráneo, produciéndose las malformaciones anatómicas características de una craneosinostosis ${ }^{4}$.

Existen diversas formas de clasificar las craneosinostosis: por la forma que adquiere el cráneo (ver más adelante), si existe solo una sutura afectada o varias (simples $v s$ complejas), cuando se asocia a otra malformación identificable (sindromática vs no sindromática), y si se logra identificar una causa subyacente (primaria $v s$ secundaria).

Existen algunos factores relacionados con mayor riesgo de presentar craneosinostosis no sindromáticas (o esporádicas). En un estudio reciente, se encontró que las gestaciones múltiples, cesárea, presentación en podálica, diabetes gestacional y oligohidroamnios se asocian a craneosinostosis ${ }^{5}$.
Se realizó una búsqueda de bibliografía en las plataformas Pubmed, Scielo y EMBASE, usando los conceptos "craneosinostosis", "plagiocefalia", "escafocefalia", "braquicefalia", seleccionando los artículos en español e inglés sobre fisiopatología y genética, clasificación, diagnóstico y derivación; optando por revisiones sistemáticas o recomendaciones de sociedades científicas cuando estuvieran disponible. Estos fueron revisados por los 3 autores que participaron en el texto. En cuando se requirió, se realizó una nueva búsqueda para responder preguntas específicas. El objetivo de esta revisión es describir las características clínicas y genéticas más frecuentes de esta patología, su calcificación y los signos más característicos para lograr reconocerla oportunamente, de manera de facilitar el diagnóstico precoz y la derivación oportuna a Neurocirugía.

\section{Fisiopatología y genética}

Las suturas son articulaciones tipo sinartrosis ${ }^{6}$. El adulto posee 16 suturas: 4 impares (metópica, sagital, coronal y lambdoidea) y 6 pareadas (escamosa, esfeno-frontal, esfeno-escamosa, esfeno-parietal, parietomastoidea y occipito-mastoidea). Están formadas por tejido fibrosos que, por un lado, evitan la separación excesiva de los huesos y por otro lado permiten el aumento de tamaño del cráneo ${ }^{7}$. El desarrollo del cerebro actúa como motor del crecimiento del cráneo.

Al cerrarse prematuramente una sutura, la expansión del cráneo se restringe en el eje perpendicular a la sutura, provocando un crecimiento compensatorio en dirección paralela a la sutura. Esto se conoce como 
Ley de Virshow y permite predecir la forma del cráneo. Además, existen otros 4 principios que explican los patrones de forma del cráneo ${ }^{8}$ :

1) Los huesos de la bóveda craneal adyacentes a la sutura sinostótica actúan como una placa ósea única, con un potencial de crecimiento reducido.

2) Se produce un depósito óseo asimétrico en las suturas de bóveda craneal y a lo largo del perímetro de la placa ósea. La deposición ósea es mayor en la periferia, al margen de la placa del hueso fusionado.

3) Debido a la restricción del crecimiento de la bóveda craneal, existe un crecimiento compensatorio que ocurre simétricamente en las suturas que están "en línea" con la sutura sinostótica. Por ej. En una la plagiocefalia anterior derecha, existe un crecimiento compensatorio en el hueso y la sutura craneal contralateral.

4) El mayor grado de crecimiento compensatorio se produce en las suturas más cercanas a la sutura fusionada, y en menor grado se produce en las suturas alejadas de la sutura afectada.

En las craneosinostosis sindromáticas (que se asocian a otras malformaciones), se pueden identificar mutaciones genéticas hasta en un 30\% de los casos ${ }^{9}$. En los pacientes en que la craneosinostosis es la única malformación identificada, estas mutaciones solo se identifican $2-5 \%$ de las veces ${ }^{10}$. Los genes más frecuentemente mutados son el Receptor del Factor de Crecimiento de Fibroblastos (FGFR1, 2 y 3) y el gen humano homólogo de Drosophila TWIST ${ }^{11}$. Estas mutaciones pueden encontrarse en familiares sanos, lo que muestra que no es el único factor causal y que existe penetrancia incompleta.

La sinostosis coronal (uni o bilateral) es la que más frecuentemente se asocia a una alteración genética. Hasta en un 30\% de los casos se encuentra una sustitución de Prolina por Arginina (Pro250arg) en el FGFR $^{12}$. Esta mutación puntual determina el síndrome de Muenke, incluso en ausencia de otras malformaciones $^{13}$.

Otras mutaciones menos frecuentes son: variantes del Receptor del Factor de Crecimiento similares a la Insulina 1 (IGF1R), el factor de transcripción RUNX2, TCF12 (el cual se encuentra en craneosinostosis no sindromáticas) y la mutación SMAD6, que se relaciona a sinostosis metópicas y sagitales ${ }^{14}$.

\section{Clasificación según forma del cráneo}

Las craneosinostosis se clasifican fenotípicamente según la forma que adquiere la cabeza al deformarse.

El cierre de la sutura sagital, provoca una cabeza que es larga en su eje anteroposterior, pero angosta en su eje lateral. Además puede presentar una prominencia en la frente o el occipucio. La fontanela anterior puede estar abierta o cerrada. Esta deformación se denomina escafocefalia (figura 1 ).

La craneosinostosis de ambas suturas coronales lleva a un cráneo corto (anteroposterior) y ancho (lateral), con la fontanela anterior abierta o cerrada. Esta forma se denomina braquicefalia (figura 2). Su diagnóstico debe llevar a la búsqueda de otras malformaciones. A su vez, el cierre de solo una de las suturas coronales, causa aplanamiento de la frente del mismo lado, con elevación del hueso esfenoides y el techo de la órbita. Habitualmente la fontanela anterior, que puede estar abierta, se desplaza contralateral a la sutura cerrada. Este tipo de malformación se denomina plagiocefalia anterior. La plagiocefalia posterior es poco frecuente, ocurre con el cierre precoz de una de las suturas lambdoideas, provocando aplanamiento occipital ipsilateral, el pabellón auricular del mismo lado es desplazado hacia posterior y hacia abajo.

El cierre de la sutura metópica, provoca una frente en quilla, con una forma triangular de la cabeza. Esta malformación se denomina trigonocefalia (figura 3). Debe diferenciarse del reborde o cresta metópica, proceso fisiológico que no se asocia a malformación del cráneo.

\section{Craneosinostosis sindromáticas}

Entre un $10 \%$ a $20 \%$ de los casos de craneosinostosis se asocian a alteración de otros órganos (craneosinostosis sindromáticas): malformación de manos y pies, cardíacas, retraso del desarrollo, entre otras, y habitualmente a más de una sutura afectada ${ }^{15,16}$. Existen más de 150 síndromes descritos que se asocian a craneosinostosis, siendo los más frecuentes y estudiados las mostrados en la tabla $1^{17}$.

Cuando existe compromiso de más de una sutura, existe mayor riesgo de complicaciones, como hipertensión endocraneana, compromiso visual y auditivo, malformación de Chiari e hidrocefalia. Habitualmente requieren varias cirugías para la corrección del defecto óseo $^{18}$.

Debe sospecharse en craneosinostosis complejas (más de una sutura afectada) o en asociación a otras malformaciones y deben ser evaluados precozmente por un equipo con experiencia en su manejo.

\section{Secuelas neurológicas y neurodesarrollo}

Se tiene claridad respecto a que en todas las craneosinostosis, inclusive en las que solo hay una sutura 
Figura 1. Escafocefalia. Imagen de paciente con escafocefalia previo a la cirugía. Destaca aumento del eje antero-posterior.

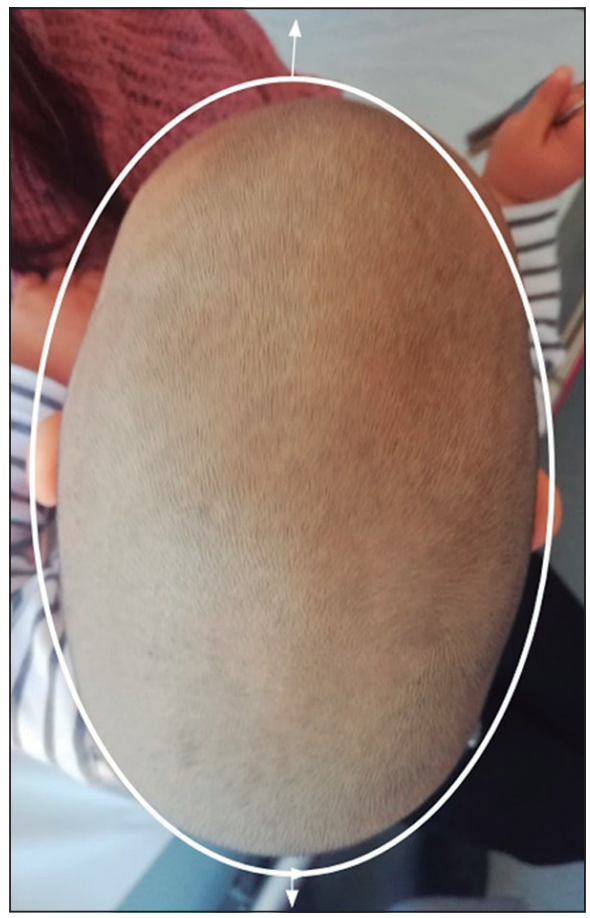

afectada, ocurre un retraso del desarrollo intelectual y motor $^{19,20}$, pero existe controversia respecto a si esta se recupera después de la cirugía ${ }^{20-24}$ incluso si se realiza a una edad precoz ${ }^{25,26}$. Solo existe evidencia poco concluyente que resolución temprana en sinostosis sagital mejora el neurodesarrollo ${ }^{27}$.

Algunos estudios con imágenes muestran que existe una compresión del tejido cortical y/o subcortical de la región adyacente a la sutura afectada ${ }^{28}$, anomalías del espacio subaracnoideo y compresión del sistema vestibular $^{29}$. Se plantea además una hipovascularización de la región próxima a la sutura afectada, lo que provoca una hipoplasia del tejido cerebral subyacente ${ }^{30}$.

La hipertensión endocraneana es frecuente y una complicación esperada en las craneosinostosis sindromáticas (hasta un 60\%) ) $^{31}$, en los pacientes con solo una sutura afectada, puede variar entre 4 a $20 \%{ }^{32}$. El trata-

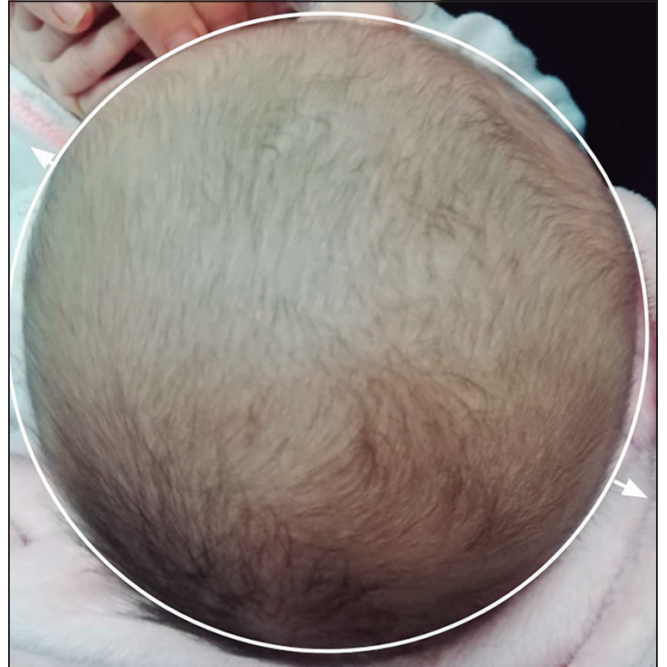

Figura 2. Braquicefalia: Paciente con braquicefalia previo a la cirugía. Destaca eje antero-posterior corto y eje lateral ancho.

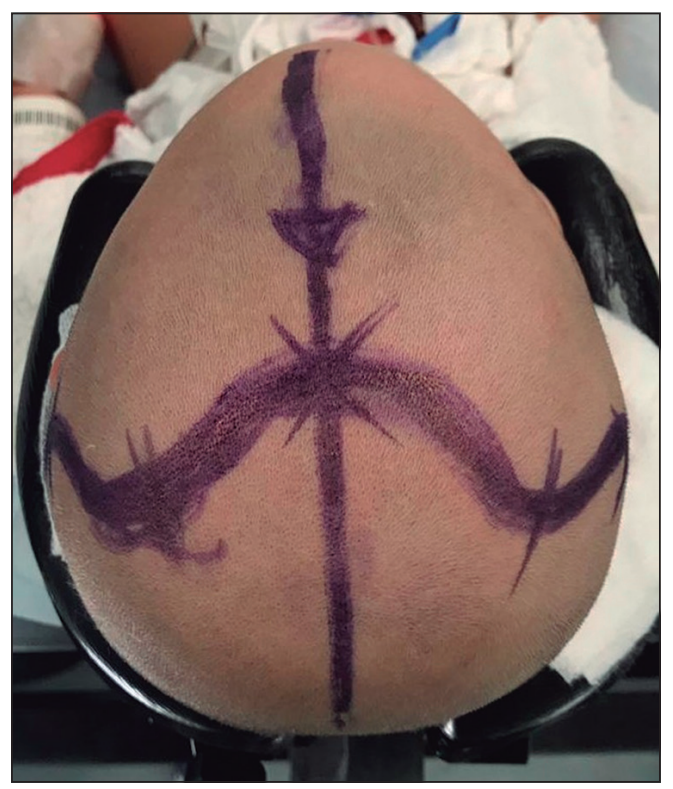

Figura 3. Trigonocefalia paciente con trigonocefalia previo a la cirugía (acostado en pabellón). Destaca forma anormal, con frente en quilla y cráneo de forma triangular.

Tabla 1. Comparación craneosinostosis sindromáticas, genes relaciones y las características clínicas. Adaptado de ${ }^{17}$

\begin{tabular}{lll}
\hline Síndrome & Genes relacionados & Características clínicas \\
\hline Apert & FGFR2 & Sindactilia de manos y pies, arco del paladar elevado, retraso del DSM \\
Crouzon & FGFR2 & Pies y manos normales, DSM normal, exoftalmo \\
Pfeiffer & FGFR2 y FGFR1 & Dedos de manos y pies anchos. Malformaciones cardiacas \\
Saethre-Chotzen & TWIST1 & Puente nasal prominente, clinodactilia, línea de implantación baja del cabello. Problemas cardiacos \\
Muenke & FGFR3 & Retraso DSM, sordera, falange media con forma de dedal \\
\hline
\end{tabular}


miento y prevención de esta es uno de los objetivos de la cirugía, junto con la mejoría estética.

\section{Plagiocefalia postural y diferenciar de craneosi- nostosis}

En el año 1992 la Asociación Americana de Pediatría (AAP) realizó una campaña de divulgación conocida como "Back to Sleep" que recomienda que los lactantes duerman en decúbito supino con el fin de disminuir el síndrome de muerte súbita. Tras esta recomendación, disminuyeron en un $40 \%$ en número de casos de muerte súbita ${ }^{33}$. Como consecuencia de esta campaña se incrementó de forma dramática el número de deformidades posturales craneales, convirtiéndose en la causa más frecuente de consulta en neurocirugía pediátrica de algunos centros, con una prevalencia de hasta $13 \%$ en niños sanos ${ }^{34}$. La plagiocefalia posterior (cierre precoz de una sutura lamboidea) es mucho menos frecuente que la deformidad posicional ${ }^{35}$.

Otras causas de plagiocefalias posturales, sin compromiso de suturas, pueden ser: conflicto de espacio intrauterino (embarazos múltiples, oligohidroamnios) o posiciones fijas en la cuna (tortícolis congénita, patología perinatal que condiciona hipomovilidad), entre otras $^{36}$.

Dentro del control sano es relevante que el médico examine la forma de la cabeza, vista por ambos lados, desde atrás y desde superior. La plagiocefalia postural es adquirida, es decir, no está presente al nacimiento; no se asocia a otras deformidades del cráneo, rostro o extremidades, y tienden a mejorar con el tiempo ${ }^{37}$. Los casos más severos o que no mejoran espontáneamente, podrían beneficiarse del uso de cascos de remodelación ${ }^{38}$.

\section{Diagnóstico}

El diagnóstico de craneosinostosis habitualmente es clínico. Se debe preguntar por factores de riesgo, tales como embarazos múltiples, oligohidroamnios y diabetes gestacional. Además, en cada control sano, el médico debe examinar la forma de la cabeza, vista por ambos lados, desde atrás y desde superior, buscando asimetrías, aplanamiento de alguna región o deformidad facial. En la vista apical del cráneo, en un paciente con plagiocefalia postural, puede verse aplanamiento del lado afectado, con el pabellón auricular y el pómulo ipsilateral adelantados. Esto provoca la forma de un paralelogramo ${ }^{37}$. En cambio, en el cierre precoz de la sutura coronal, se produce aplanamiento de la región occipital y frontal del lado afectado, dando una forma trapezoidal. En el cierre de la sutura lambdoidea puede deformarse la cabeza como paralelogramo, pero el pabellón auricular estará desplazado hacia atrás y abajo ${ }^{39}$.

Se debe palpar buscando simetría de las suturas y la forma del bregma. El cierre precoz de la fontanela anterior es un signo poco sensible de craneosinostosis, siendo más relevante la asimetría del cráneo. El cierre de una única sutura no afecta la circunferencia craneana precozmente, por lo que una circunferencia de cráneo normal no descarta el diagnóstico.

Si el pediatra sospecha una plagiocefalia postural (una asimetría que no estaba presente al nacimiento, en zona de apoyo) puede solicitar una radiografía de cráneo (anteroposterior, lateral de ambos lados y Townes) para evaluar la presencia de suturas ${ }^{40}$. Si se observan todas las suturas, es posible indicar cambios en la posición de la cabeza al dormir y pasar algún tiempo apoyados en el abdomen (estando despierto y observado) y control clínico posterior. En caso de asimetría craneal presente desde el nacimiento o que va progresando, o una radiografía no concluyente, debe derivarse precozmente a un centro que cuente con neurocirugía. El TC de cráneo es el Gold Standart para el diagnóstico y planificación de la cirugía. Existen algunos estudios que avalan la ecografía de suturas ${ }^{41}$, pero estos autores no la recomiendan, por ser operador dependiente y con baja sensibilidad, lo que podría retrasar el diagnóstico y resolución quirúrgica.

\section{Manejo quirúrgico}

Investigaciones recientes han demostrado que el tratamiento quirúrgico de la craneosinostosis resulta en mejoría, no solo la patología de la bóveda craneal, sino también en las anormalidades de la base del cráneo e incluso las deformidades faciales que se desarrollan de forma secundaria a las restricciones del crecimiento por la sutura sinostótica ${ }^{42}$.

Las indicaciones principales para el tratamiento quirúrgico de la craneosinostosis son: evitar el aumento de la presión intracraneal y solucionar la forma anormal del cráneo. El hecho de mejorar el volumen craneal previene las secuelas del aumento de la PIC, como los trastornos en el desarrollo neurológico, la discapacidad intelectual, y el compromiso visual ${ }^{43}$.

Las técnicas quirúrgicas se pueden dividir en dos grandes grupos:

- Las técnicas osteoclásticas (suturectomía): se refiere a la resección de la sutura sinostótica, puede realizarse en forma convencional o asistida por endoscopia. La suturectomía asociada al uso de dispositivos de distracción ósea y cascos que apoyan la remodelación del cráneo y permite que el cerebro en desarrollo y en expansión cambie la forma 
del cráneo. Estas técnicas se basan en la capacidad de autocorrección del cráneo y el encéfalo, y eliminan el impulso (sinostosis) adverso a la dirección de crecimiento del cráneo.

- Las técnicas de remodelación (por ej. avance fronto-orbitario), por otro lado, intentan obtener la forma deseada del cráneo mediante una reconstrucción completa. Necesitan habitualmente de varias intervenciones quirúrgicas, según el tipo de paciente y requieren un equipo de cirugía craneofacial con experiencia ${ }^{44}$.

La técnica de reconstrucción quirúrgica debe adaptarse al tipo de sinostosis y la edad del paciente. La remodelación en un niño menor de 1 año se logra más fácilmente sin una alteración ósea significativa que en un niño mayor. La calidad de los huesos de la bóveda craneal difiere según la edad del niño. En general, los niños de menos de 6 meses tienen huesos que están mucho menos mineralizados y, por lo tanto, menos quebradizos. Esto provee una plasticidad adicional y permite un contorneado más fácil de los huesos ${ }^{45}$.

La cirugía precoz (idealmente antes del 1 año de vida) previene la aparición de hipertensión endocraneana, permite realizar procedimientos menos invasivos y suele tener mejor pronóstico estético que la cirugía tardía ${ }^{46-48}$.

Con respecto a las complicaciones, en la craneosinostosis no sindromática la incidencia de infección postoperatoria es bastante baja, que varían entre el $2,5 \%$ hasta el $6,5 \%{ }^{49}$. En las craneosinostosis sindromáticas, la incidencia de 3,2\% a 10,4\% en los casos complejos $^{50}$. Entre los factores influyentes para infecciones de la herida quirúrgica destacan la duración de la cirugía, la edad del paciente, la combinación de intervención intracraneal y extracraneal, y número de cirujanos presentes operando. La mortalidad es muy baja para todos los procedimientos, del $0 \%$ al $1 \%{ }^{51}$.

\section{Conclusión y recomendaciones}

La craneosinostosis es una patología poco frecuente, que debe sospecharse cuando se observa una alteración en la forma del cráneo. En ocasiones se asocia a otras malformaciones, sobre todo en las craneosinostosis complejas. Puede asociarse a compromiso neurológico, retraso del desarrollo, hipertensión endocraneana y alteraciones de la vía visual. La derivación precoz (idealmente antes de los 6 meses) permite realizar una cirugía temprana, menos invasiva y con menor morbilidad, disminuyendo las complicaciones estéticas y neurológicas.

Por lo tanto, como autores recomendamos derivar precozmente a Neurocirugía a los lactantes con asimetría craneana presente desde el nacimiento y/o que va aumentando en el tiempo. Al contrario, la asimetría de cráneo adquirida (no presente al nacimiento), en zona de apoyo, sin otras malformaciones faciales o en extremidades y que va mejorando con el tiempo, puede solicitarse radiografía de cráneo y control clínico posterior.

Una fontanela anterior no palpable, sin retraso del desarrollo psicomotor, sin alteración del perímetro craneano, en un cráneo simétrico, es poco sugerente de craneosinostosis. Una fontanela anterior abierta y una circunferencia de cráneo normal, no descarta el cierre precoz de una sutura en un paciente con asimetría craneal.

\section{Conflicto de intereses}

Los autores declaran no tener conflicto de intereses.

\section{Referencias}

1. Governale LS. Craniosynostosis. Pediatr Neurol 2015;53:394-401. https://doi. org/10.1016/j.pediatrneurol.2015.07.006.

2. Zuleta F. A. Craneosinostosis: Visión del neurocirujano. Rev Chil Pediatría 2002;73:506-7. https://doi.org/10.4067/ S0370-41062002000500011.

3. Rocco FD, Arnaud E, Renier D. Evolution in the frequency of nonsyndromic craniosynostosis: Clinical article. J Neurosurg Pediatr 2009;4:21-5. https:// doi.org/10.3171/2009.3.PEDS08355.

4. Abd-El-Barr MM. Molecular Genetics and Principles of Craniosynostosis. En Youmans and Winn ed. Neurol. Surg. 7th ed., Elsevier; n.d., p. 1550\uc0 $\backslash u 8211\{\} 5$.

5. Sergesketter AR, Elsamadicy AA, Lubkin
DT, Krucoff KB, Krucoff MO, Muh CR. Characterization of Perinatal Risk Factors and Complications Associated with Nonsyndromic Craniosynostosis. J Craniofac Surg 2018;34-8. https://doi. org/10.1097/SCS.0000000000004997.

6. Di Ieva A, Bruner E, Davidson J, et al. Cranial sutures: a multidisciplinary review. Childs Nerv Syst 2013;29:893-905. https://doi.org/10.1007/s00381-013-20614.

7. Raam MS, Solomon BD, Shalev SA, Muenke M. Holoprosencephaly and craniosynostosis: A report of two siblings and review of the literature. Am J Med Genet C Semin Med Genet 2010;154C:176-82. https://doi. org/10.1002/ajmg.c.30234.

8. Lin KY, Persing JA. Molecular Genetics and Principles of Craniosynostosis. En Youmans and Winn ed. Neurol. Surg. 7th ed., Elsevier; n.d., p. 1556-69.

9. Wilkie AOM, Johnson D, Wall SA. Clinical genetics of craniosynostosis. Curr Opin Pediatr 2017;29:622-8. https://doi. org/10.1097/MOP.0000000000000542.

10. Wilkie AOM, Byren JC, Hurst JA, et al. Prevalence and complications of single-gene and chromosomal disorders in craniosynostosis. Pediatrics 2010;126:e391-400. https://doi. org/10.1542/peds.2009-3491.

11. Lattanzi W, Bukvic N, Barba M, et al. Genetic basis of single-suture synostoses: genes, chromosomes and clinical implications. Childs Nerv Syst 2012;28:1301-10. https://doi.org/10.1007/ s00381-012-1781-1. 
12. Moloney DM, Wall SA, Ashworth GJ, et al. Prevalence of Pro250Arg mutation of fibroblast growth factor receptor 3 in coronal craniosynostosis. Lancet 1997;349:1059-62. https://doi. org/10.1016/s0140-6736(96)09082-4.

13. Muenke M, Gripp KW, McDonaldMcGinn DM, et al. A unique point mutation in the fibroblast growth factor receptor 3 gene (FGFR3) defines a new craniosynostosis syndrome. Am J Hum Genet 1997;60:555-64.

14. Sharma VP, Fenwick AL, Brockop MS, et al. Mutations in TCF12, encoding a basic helix-loop-helix partner of TWIST1, are a frequent cause of coronal craniosynostosis. Nat Genet 2013;45:3047. https://doi.org/10.1038/ng.2531.

15. Armand T, Schaefer E, Di Rocco F, Edery P, Collet C, Rossi M. Genetic bases of craniosynostoses: An update. Neurochirurgie 2019;65:196-201. https:// doi.org/10.1016/j.neuchi.2019.10.003.

16. Wang JC, Nagy L, Demke JC. Syndromic Craniosynostosis. Facial Plast Surg Clin N Am 2016;24:531-43. https://doi. org/10.1016/j.fsc.2016.06.008.

17. Sawh-Martínez R, Steinbacher DM. Syndromic Craniosynostosis. Clin Plast Surg 2019;46:141-55. https://doi. org/10.1016/j.cps.2018.11.009.

18. Nagy L, Demke JC. Craniofacial anomalies. Facial Plast Surg Clin N Am 2014;22:523-48. https://doi.org/10.1016/j. fsc.2014.08.002.

19. Speltz ML, Collett BR, Wallace ER, et al. Intellectual and academic functioning of school-age children with singlesuture craniosynostosis. Pediatrics 2015;135:e615-623. https://doi. org/10.1542/peds.2014-1634.

20. Da Costa AC, Walters I, Savarirayan $\mathrm{R}$, Anderson VA, Wrennall JA, Meara JG. Intellectual outcomes in children and adolescents with syndromic and nonsyndromic craniosynostosis. Plast Reconstr Surg 2006;118:17581; discussion 182-183. https://doi. org/10.1097/01.prs.0000221009.93022.50.

21. Cohen SR, Cho DC, Nichols SL, Simms C, Cross KP, Burstein FD. American society of maxillofacial surgeons outcome study: preoperative and postoperative neurodevelopmental findings in single-suture craniosynostosis. Plast Reconstr Surg 2004;114:8417. https://doi.org/10.1097/01. prs.0000132854.14237.a8.

22. Nejad EA, Nikoobakhat M. Postoperative neurodevelopmental findings in syndromic craniosynostosis. Iran J Child Neurol 2010;3:45-50. https://doi. org/10.22037/ijcn.v3i4.1537.

23. Speltz ML, Kapp-Simon K, Collett B, et al. Neurodevelopment of infants with single-suture craniosynostosis: presurgery comparisons with case-matched controls. Plast Reconstr Surg 2007;119:187481. https://doi.org/10.1097/01. prs.0000259184.88265.3f.

24. Da Costa AC, Anderson VA, Savarirayan R, et al. Neurodevelopmental functioning of infants with untreated single-suture craniosynostosis during early infancy. Childs Nerv Syst 2012;28:869-77. https://doi.org/10.1007/ s00381-011-1660-1.

25. Starr JR, Kapp-Simon KA, Cloonan YK, et al. Presurgical and postsurgical assessment of the neurodevelopment of infants with single-suture craniosynostosis: comparison with controls. J Neurosurg 2007;107:103-10. https://doi.org/10.3171/ PED-07/08/103.

26. Toth K, Collett B, Kapp-Simon KA, et al. Memory and response inhibition in young children with single-suture craniosynostosis. Child Neuropsychol J Norm Abnorm Dev Child Adolesc 2008;14:339-52. https://doi. org/10.1080/09297040701594888.

27. Mandela R, Bellew M, Chumas $P$, Nash H. Impact of surgery timing for craniosynostosis on neurodevelopmental outcomes: a systematic review. J Neurosurg Pediatr 2019;23:442-54. https://doi.org/10.3171/2018.10. PEDS18536.

28. Magge KT, Magge SN, Keating RF, et al. Incidental findings on preoperative computed tomography for nonsyndromic single suture craniosynostosis. J Craniofac Surg 2014;25:1327-30. https://doi. org/10.1097/SCS.0000000000000797.

29. Carmel PW, Luken MG, Ascherl GF. Craniosynostosis: computed tomographic evaluation of skull base and calvarial deformities and associated intracranial changes. Neurosurgery 1981;9:366-72.

30. Cohen SR, Persing JA. Intracranial pressure in single-suture craniosynostosis. Cleft Palate-Craniofacial J Off Publ Am Cleft Palate-Craniofacial Assoc 1998;35:194-6. https://doi. org/10.1597/1545-1569_1998_035_0194_ ipissc_2.3.co_2.

31. Lumenta CB, Di Rocco C, Haase J, Mooij JJA eds: Neurosurgery. En Kehr PH, Kaech D. ed: European manual of medicine: vol. 23. 2013.

32. Tamburrini G, Caldarelli M, Massimi L, Santini P, Di Rocco C. Intracranial pressure monitoring in children with single suture and complex craniosynostosis: a review. Childs Nerv Syst 2005;21:913-21. https://doi. org/10.1007/s00381-004-1117-x.

33. Positioning and sudden infant death syndrome (SIDS): update. American Academy of Pediatrics Task Force on Infant Positioning and SIDS. Pediatrics 1996;98:1216-8.
34. Littlefield TR, Saba NM, Kelly KM. On the current incidence of deformational plagiocephaly: an estimation based on prospective registration at a single center. Semin Pediatr Neurol 2004;11:301-4. https://doi.org/10.1016/j. spen.2004.10.003.

35. Davis BE, Moon RY, Sachs HC, Ottolini MC. Effects of sleep position on infant motor development. Pediatrics 1998;102:1135-40. https://doi. org/10.1542/peds.102.5.1135.

36. Rekate HL. Occipital plagiocephaly: a critical review of the literature. J Neurosurg 1998;89:24-30. https://doi. org/10.3171/jns.1998.89.1.0024.

37. Laughlin J, Luerssen TG, Dias MS, Committee on Practice and Ambulatory Medicine, Section on Neurological Surgery. Prevention and management of positional skull deformities in infants. Pediatrics 2011;128:1236-41. https://doi. org/10.1542/peds.2011-2220.

38. Dec W, Warren SM. Current Concepts in Deformational Plagiocephaly: J Craniofac Surg 2011;22:6-8. https://doi.org/10.1097/ SCS.0b013e3182074e04.

39. Levi B, Wan DC, Longaker MT, Habal MB. Deformational Plagiocephaly: A Look Into the Future. J Craniofac Surg 2011;22:3-5. https://doi.org/10.1097/ SCS.0b013e3181fb7ee5.

40. Mazzola C, Baird LC, Bauer DF, et al. Congress of Neurological Surgeons Systematic Review and EvidenceBased Guideline for the Diagnosis of Patients With Positional Plagiocephaly: The Role of Imaging. Neurosurgery 2016;79:E625-6. https://doi.org/10.1227/ NEU.0000000000001427.

41. Rozovsky K, Udjus K, Wilson N, Barrowman NJ, Simanovsky N, Miller E. Cranial Ultrasound as a First-Line Imaging Examination for Craniosynostosis. Pediatrics 2016;137:e20152230. https://doi. org/10.1542/peds.2015-2230.

42. Di Rocco F, Renier D, Marchac D. Syndromic Craniosynostosis. En Youmans and Winn de. Neurol. Surg. 7th ed., Elsevier; n.d., p. 15561569.

43. Christian EA, Imahiyerobo TA, Nallapa S, Urata M, McComb JG, Krieger MD. Intracranial hypertension after surgical correction for craniosynostosis: a systematic review. Neurosurg Focus 2015;38:E6. https://doi. org/10.3171/2015.2.FOCUS14853.

44. Rivero-Garvia M. Craneosinotosis complejas. En Orduna Martínez J, López Pison J. ed. Neurocirugía pediátrica: fundamentos de patología neuroquirúrgica para pediatras. Madrid: Ergon; 2017.

45. Jiménez DF, McGinity MJ, Barone CM. Endoscopy-assisted early correction of 
single-suture metopic craniosynostosis: a 19-year experience. J Neurosurg Pediatr 2019;23:61-74. https://doi. org/10.3171/2018.6.PEDS1749.

46. Taylor JA, Maugans TA. Comparison of spring-mediated cranioplasty to minimally invasive strip craniectomy and barrel staving for early treatment of sagittal craniosynostosis. J Craniofac Surg 2011;22:1225-9. https://doi.org/10.1097/ SCS.0b013e31821c0f10.

47. Rottgers SA, Kim PD, Kumar AR, Cray JJ, Losee JE, Pollack IF.
Cranial vault remodeling for sagittal craniosynostosis in older children. Neurosurg Focus 2011;31:E3. https://doi. org/10.3171/2011.5.FOCUS1196.

48. Jiménez DF, Barone CM. Endoscopic technique for sagittal synostosis. Childs Nerv Syst 2012;28:1333-9. https://doi. org/10.1007/s00381-012-1768-y.

49. Shastin D, Peacock S, Guruswamy $\mathrm{V}$, et al. A proposal for a new classification of complications in craniosynostosis surgery. J Neurosurg Pediatr 2017;19:675-83. https://doi.
org/10.3171/2017.1.PEDS16343.

50. Rottgers SA, Lohani S, Proctor MR Outcomes of endoscopic suturectomy with postoperative helmet therapy in bilateral coronal craniosynostosis. J Neurosurg Pediatr 2016;18:281-6. https:// doi.org/10.3171/2016.2.PEDS15693.

51. Mathijssen IMJ. Guideline for Care of Patients With the Diagnoses of Craniosynostosis: Working Group on Craniosynostosis. J Craniofac Surg 2015;26:1735-807. https://doi. org/10.1097/SCS.0000000000002016. 